\title{
Indoor Map aided INS/Wi-Fi integrated LBS on Smartphone Platforms
}

\author{
Chunyang $\mathrm{Yu}^{\mathrm{a}}$, Naser El-Sheimy ${ }^{\mathrm{a}}$ \\ ${ }^{a}$ Geomatics Engineering, University of Calgary, 2500 University Dr NW, Calgary, AB T2N 1N4, Canada \\ chunyang.yu@ucalgary.ca; \\ elsheimy@ucalgary.ca
}

\begin{abstract}
KEY WORDS: Indoor Location-Based Service (LBS), Wi-Fi-based positioning, Inertial Navigation System (INS), map-aiding indoor position, low-cost Microelectromechanical systems (MEMS) Sensors devices, integrated indoor position method
\end{abstract}

\begin{abstract}
:
In this research, an indoor map aided INS/Wi-Fi integrated location based services (LBS) applications is proposed and implemented on smartphone platforms. Indoor map information together with measurements from an inertial measurement unit (IMU) and Received Signal Strength Indicator (RSSI) value from Wi-Fi are collected to obtain an accurate, continuous, and low-cost position solution. The main challenge of this research is to make effective use of various measurements that complement each other without increasing the computational burden of the system. The integrated system in this paper includes three modules: INS, Wi-Fi (if signal available) and indoor maps. A cascade structure Particle/Kalman filter framework is applied to combine the different modules. Firstly, INS position and Wi-Fi fingerprint position integrated through Kalman filter for estimating positioning information. Then, indoor map information is applied to correct the error of INS/Wi-Fi estimated position through particle filter. Indoor tests show that the proposed method can effectively reduce the accumulation positioning errors of stand-alone INS systems, and provide stable, continuous and reliable indoor location service.
\end{abstract}

\section{INTRODUCTION}

Indoor Location-Based Services (LBS), which is the foundation of positioning-based for big data and the internet of things, become increasingly important in many of our day-to-day activities (Junglas, A., 2008). A report released by Markets and Markets indicates that LBS market is expected to grow from 15.04 billion to $\$ 77.84$ billion by 2021 , at a compound annual growth rate of $38.9 \%$ (Marketsandmarkets, 2016). LBS can be used in various kinds of applications, such as enterprise services, mobile advertisements and location-based Business Intelligence (BI). Therefore, numerous high-tech companies and research institutions, e.g. Microsoft, Google, Intel and Apple, are focusing on the research and development of LBS applications and systems.

Currently, the main techniques for indoor LBS includes Wi-Fi based positioning, blue-tooth positioning, inertial sensor based positioning, and magnetic-based positioning (Asghar, Dr, Muhammad Zubair, 2014). The use of wireless signals, such as $\mathrm{Wi}-\mathrm{Fi}$ and blue-tooth, for indoors navigation gain momentum with the advent of personal wireless communication technologies, the continuous improvements in microelectronics, and the wide adaption of personal communication devices. However, these methods require enough $\mathrm{Wi}-\mathrm{Fi}$ access points or i-Beacons which cost considerable time and money to install and maintain. Furthermore, it is sensitive to environment disturbance.

For non-infrastructure-based techniques, especially inertial navigation system, it is self-contained and independent, and not affected by the environment (Lan, Haiyu, 2015). Additionally, Microelectromechanical systems (MEMS) Sensors devices are convenient and low-cost, and have infiltrated our daily live, e.g., smartphone, smart watch, smart sports bracelet. However, the error of the derived position from MEMS sensors grow very quickly with time because of the fundamental process of integration processes of their signals (Naser, El-Sheimy ,2012).
Therefore, aiding sensors or information are needed to provide an accurate, continuous, and stable position solution for indoor LBS.

For commercial LBS, one of the first considerations is the cost, the computational burden and the time consumption. Nowadays, digital maps can be easily downloaded through scanning Quick Response (QR) codes or internet using smart-devices. Moreover, no additional infrastructure is needed for map aiding position. Furthermore, considering the wide, increasing coverage of free wireless Wi-Fi in public areas (Zanella, Andrea, 2014), Wi-Fi based positioning, which only uses preexisting Wi-Fi infrastructures, is used as another potential aiding technology for indoor position. Considering the above issues, in this research, a map aided INS/Wi-Fi integrated indoor position method is proposed.

As shown in Figure 1, there are three modules used in this research: INS module, Wi-Fi module (if signal available) and map module. If Wi-Fi signal is available, inertial positon result and Wi-Fi derived position through using fingerprinting method are integrated through extended Kalman filter to correct the INS derived position (CY, Yu, 2017). Then, map information is applied to aid the estimated position through map aiding method on a particle filter platform. Moreover, the system without WiFi signal can still satisfy the requirement of indoor LBS. 


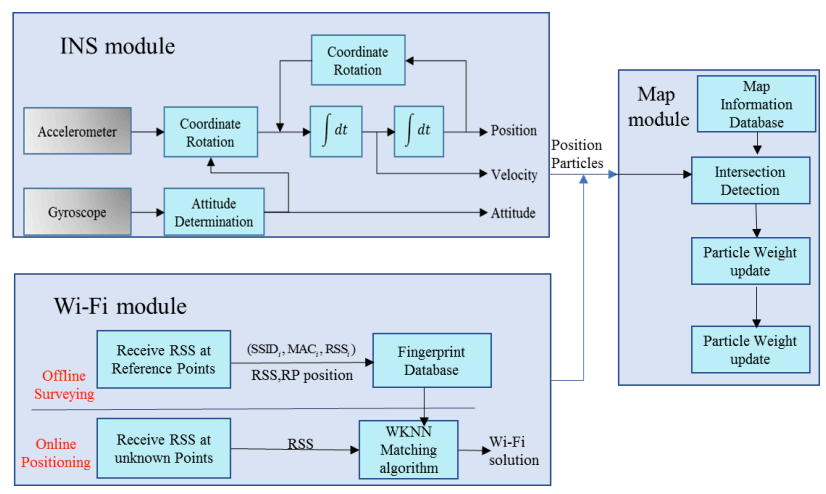

Figure 1. Algorithm structure

\section{MAIN ALGORITHM}

The paper will present the location algorithm of each module and the integration algorithm between them. Specifically, the strapdown inertial sensor (SINS) algorithm and the fingerprinting method are used to calculate the basic location information and the auxiliary Wi-Fi derived position in INS module and Wi-Fi module. Map Aiding method is applied in the map module to aid the map information into the LBS. Additionally, a Kalman/Particle filter cascade structure is proposed to add the map constrain to the INS/Wi-Fi integrated solution, which can increase the LBS accuracy without adding high computational burden.

\subsection{INS module}

INS combines the measurements of accelerometers and gyroscopes to continuously track a moving object's motion. The position, velocity and attitude of this object relative to a known initial point, velocity and orientation can be calculated through SINS method.

The INS module, shown in Figure 1, gives an overview of the SINS algorithm, the angular velocity is used to calculate the attitude of the MEMS-IMU relative to the navigation frame, then the specific force measurements from the accelerometers are projected into navigation frame using the calculated attitude. Acceleration due to gravity is then compensted to obtain the acceleration of the MEMS-IMU in the navigation frame. This acceleration is then integrated once to estimate the velocity of the device and again to estimate its position. For this indoor LBS, the SINS algorithm is used to obtain the primary position information. However, the estimation error from INS grows very quickly with time because of the integration processes. Therefore, aiding sensors or information are needed to provide an accurate, continuous, and stable positioning solution for indoor LBS (Cho, S. Y. 2006).

Non-holonomic constraint (NHC) is one of the most commonly used auxiliary navigation information for INS (Niu X, 2013). The assumption in NHC is that a moving platform cannot skid or jump, therefore the lateral and vertical speeds can be assumed to be zeroes. The forward, lateral, and vertical speeds work as the velocity update for the INS to limit the INS velocity errors through EKF.

\subsection{Wi-Fi module}

Fingerprinting and trilateration are two popular Wi-Fi based positioning methods, and each of them has their advantages and disadvantages. In our research, the fingerprinting method is used because it can provide position without any knowledge of AP location or signal-propagation model. As showed in Figure 2, fingerprint-based Wi-Fi positioning usually has two operating steps: (1) the offline pre-survey and (2) the online positioning (Bahl, Paramvir, 2000).

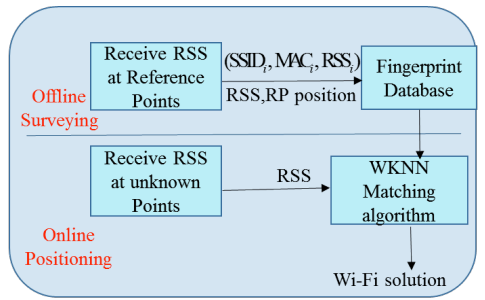

Figure 2. Fingerprinting-based Wi-Fi positioning Algorithm

In the first step, RRSI from available APs and position information are collected as fingerprints to populate a database (Stella, Maja, 2014). Each fingerprint in the radio-map database is recorded as a vector which normally has the following form, $\mathrm{S}_{i}=\left\{\left(\mathrm{SSID}_{1}, \mathrm{MAC}_{1}, \mathrm{RSS}_{1}\right), \cdots\left(\mathrm{SSID}_{\mathrm{n}}, \mathrm{MAC}_{\mathrm{n}}, \mathrm{RSS}_{\mathrm{n}}\right)\right\} \quad . \quad$ In which, SSID and MAC respectively present the name and the Mac address of a specific AP (CY, Yu, 2017).

In the second step, the device's position is estimated by comparing the measured vector of RSS with the fingerprints in the pre-built database in step one, and then the closest fingerprints are determined (Haiyu Lan, 2015). The Weighted K-Nearest Neighbor (WKNN) algorithm is used to get the optimal match between the device's newly-collected RSSs and those RSSs stored in the radio-map database. The device's current estimated position can be updated by weighting the corresponding $\mathrm{K}$ reference coordinates (fingerprints) in the radio-map database using the following equation:

$$
\hat{p}=\sum_{j=1}^{k}\left(\frac{1 / \mathrm{d}_{j}}{\sum_{j=1}^{k}\left(1 / \mathrm{d}_{j}\right)} p_{j}\right)
$$

where $\hat{p}$ is the estimated navigation coordinate; and $p_{j}(j=1,2, \cdots, k)$ is the position of the $\mathrm{j}$-th selected reference coordinates in the database. $\mathrm{d}=\left[\mathrm{d}_{1} \cdots \mathrm{d}_{m}\right]$, in which $\mathrm{d}_{i}$ is the two-dimensional Euclidean distance between $\alpha_{i}$ and $\beta . \mathrm{d}_{i}$ is defined as $\mathrm{d}_{i}=\left|\alpha_{i}-\beta\right|, \quad(i=1,2, \cdots, m) \cdot \alpha=\left\{\alpha_{1}, \alpha_{2}, \ldots, \alpha_{m}\right\}$ represents the RSS set of the database's fingerprints. The measured RSS values from $n$ APs at an unknown location are expressed as a set $\beta=\left\{\beta_{1}, \beta_{2}, \ldots, \beta_{n}\right\} . \mathrm{K}$ is the setting number of the K-nearest neighbours (points) with smallest distances to $\beta$.

\subsection{Map module}

Indoor map information is used to constrain the primary INS solution through the particle filter (PF). PF was invented to numerically implement the Bayesian estimator, and a set of particles samples are used to represent the posterior density through Monte Carlo approach (Gustafsson Fredrik, 2010):

$$
p\left(x_{t} \mid \mathrm{Y}_{t}\right) \approx \sum_{i=1}^{N} w_{t}^{(i)} \delta\left(x-x_{t}^{(i)}\right)
$$

In which $w_{t}^{(i)}$ is the $\mathrm{i}$-th particle's weight at time t. The mean value of the sample is used to get the minimum variance of the states. 


$$
E\left[f\left(x_{k}\right)\right] \approx \sum_{i=1}^{N} q_{k}^{i} f\left(x_{k}^{i}\right)
$$

In this research, Auxiliary Particle filter (APF) is applied instead of the traditional particle filter. Because the latter selects the state transition probability density function as importance of distribution function, which did not take the measurements into consideration, and might cause unsatisfactory sampling results. This will definitely lead to the situation where the weights of many particles are zero or close to zero, and just a few number of particles are duplicated during the resampling processes, that is, the PF method loses its diversity, and suffers from particle impoverishment, and different kinds of PF has already been test in our previous publication (Chunyang $\mathrm{Yu}, 2016$ ).

Different from the traditional PF, APF previously tests the current particles to determine the effective particles in next step, and deletes the current particles who do not match the current measurement. It takes the measurement into consideration and provide an efficient way to solve the particle impoverishment problem. There are three phases in APF, system propagation, measurement update and resampling (if it is needed).

The map information is used at the measurement update phase of APF through wall-cross method (Cossaboom M, 2012). As shown in Figure 3, in the measurement update process, twodimension indoor architectural map information is used as new measurement to update the weight of the particles (Grisetti G, 2007). To be specific, after system propagation, if the new particle has intersection with the wall, then this particle is invalid and the weight of this particle will be assigned as zero. If a wall is not intersected by the particle during the propagation step, which means the new generated particle is effective, then the weight of the particle remains the same with the previous step.

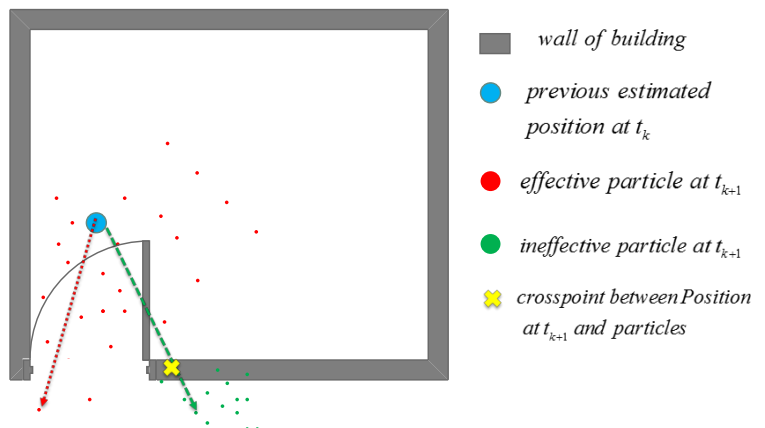

Figure 3. Fingerprinting-based Wi-Fi positioning Algorithm

\subsection{Two-layer Structure:}

The main challenge of this research is to combine different kinds of measurements and information to obtain an accurate, low-cost and continuous indoor positioning solution with low computational requirement for mobile devices. Therefore, a two-layer Kalman/Particle filter structure algorithm is proposed. Figure 4 shows the algorithm structure of the paper.

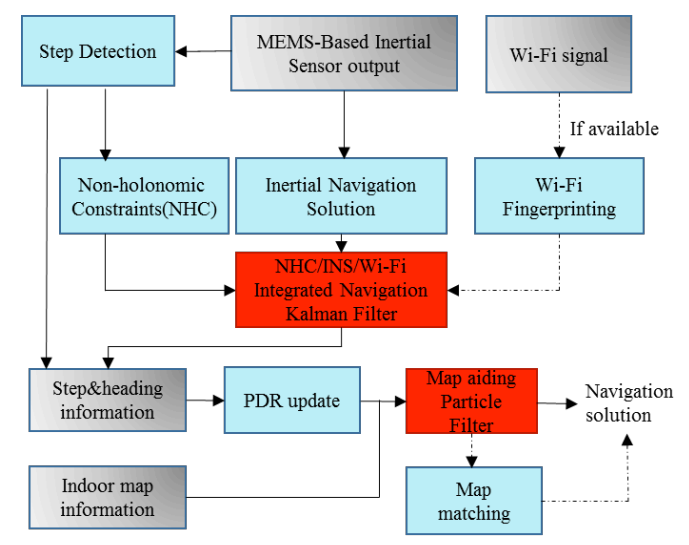

Figure 4. Map/INS/Wi-Fi integration algorithm using KF and PF

The KF is used on the bottom layer of the algorithm, and the update cycle is according to the high rate inertial sensors measurements. KF can overcome implementation issues of PF, such as computational burden, particle degeneracy. NonHolonomic constraints and $\mathrm{Wi}-\mathrm{Fi}$ estimated position (if $\mathrm{Wi}-\mathrm{Fi}$ is available) are used as inputs of KF to correct the preliminary INS positioning solution.

The step and heading information calculated from the primary Wi-Fi/INS solution are utilized to perform the update of upper particle filter, and the update rate is the same as the low step detection rate. In this research, step detection of the upper filter is performed during the INS mechanization process in the lower filter by using the method in (Cho, S. Y, 2006). The stride length and heading estimation result derived from lower Kalman filter are the measurements of the upper PF.

On the upper layer of the algorithm, the APF is used to introduce map information to the primary $\mathrm{Wi}-\mathrm{Fi} / \mathrm{INS}$ integrated solution. Particle filter is easy and convenient for adding additional map information, and it can accommodate arbitrary sensor characteristics, motion dynamics, and noise distributions. Moreover, the number of samples used in PF can be controlled to suit the available computational resources, especially in some enhanced versions of PF. The Dead Reckoning (DR) positioning technique is used to build the system APF update model. After that indoor map information is used as new measurement to re-set the weights of particles and get a map constrained positioning solution.

\section{TEST AND ANALYSIS}

\subsection{Test}

The validity and feasibility of the proposed algorithm are confirmed through real experiments. The experiemnets took place on the first floor of the Energy Environment Experiential Learning (EEEL) building at the University of Calgary, and the time duration is about 25 minutes.

Figure 5 shows the experiment environment, and it is clear from the figure that there is a hall in this building. Therefeore, there is not enough architecture map information that can be used to provide constrains to the INS derived solution. Figure 6 is the designed experiment trajectory plotted on the two-dimension digital map of the first floor, square 112 in Figure 6 corresponds to the hall area in Figure 5. 


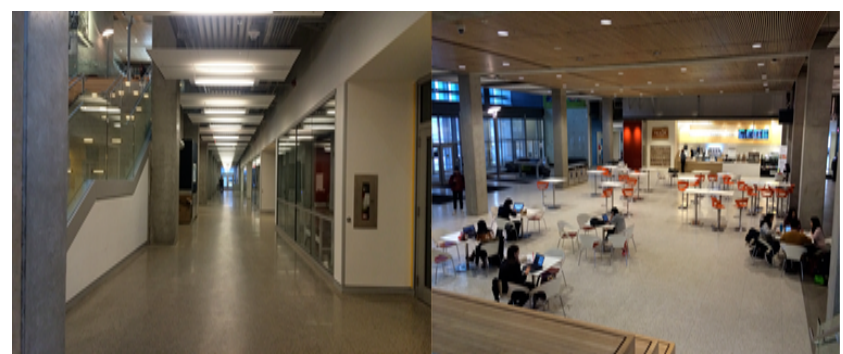

Figure 5 experiment environment in EEEL building

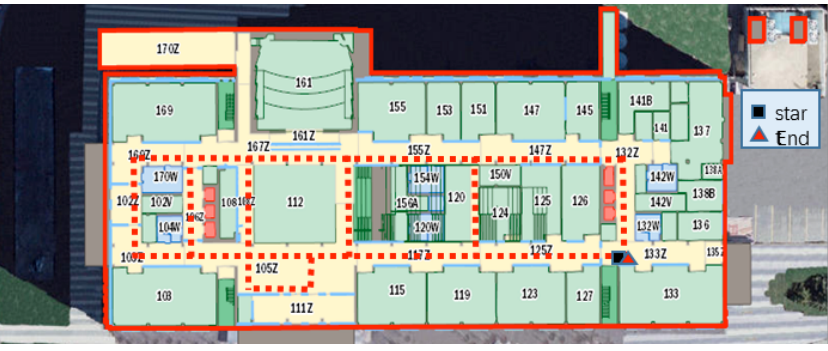

Figure 6 Designed trajectory for the test

The proposed algorithm has been implemented on the Samsung Galaxy Note 4 smartphone. The sensors' type of the device is shown in Table 1.

Table 1. Sensors of Samsung S4

\begin{tabular}{cc}
\hline Sensor & Samsung S4 \\
\cline { 2 - 2 } & Model \\
\hline Accelerometer & STM K3DH \\
\hline Gyroscope & STM K3G \\
\hline Compass & AKM AK8975 \\
\hline Barometer & Bosch BMP182
\end{tabular}

\subsection{Result}

Figure 7 is the reference trajectory of the test. Figure 8 shows the INS derived position without auxiliary Wi-Fi position and map information. If we compare the INS-derived solution with the reference trajectory, it is obvious that INS cannot provide a satisfying estimated position even with NHC constrains. Due to the standalone low-cost MEMS based INS, when velocity is used to correct the system error, the heading of the system is unobservable, which is also confirmed in the observability analysis conducted in (Zhang, X, 2012). Therefore, aiding information is needed to further correct the error of the system.

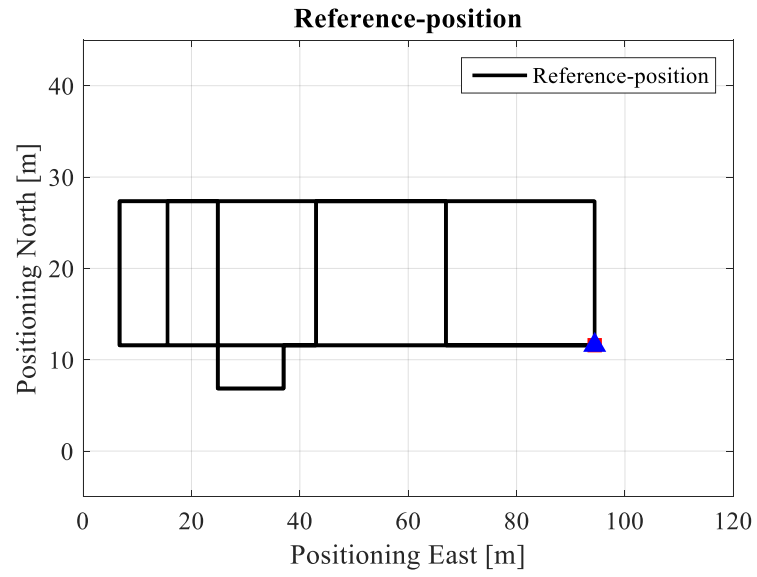

Figure 7. Reference trajectory of the test

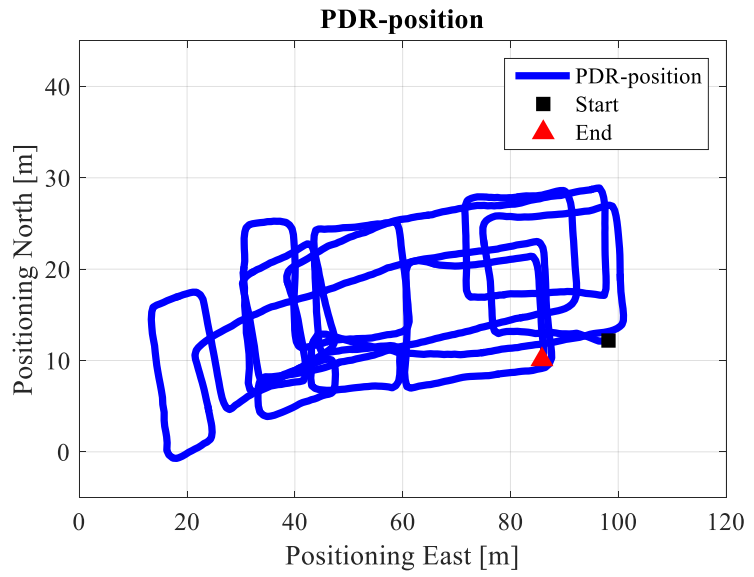

Figure 8. Reference trajectory of the test

Figure 9 shows the Wi-Fi fingerprint estimated position, and Figure 10 is the INS/Wi-Fi integrated position. We can see that there are jumping points in the Wi-Fi Fingerprint solution. However, when we aid the Wi-Fi position into the system, it is clear to see that the integrated algorithm can complement each method and provide a better lower filter solution, but there is still a big drift in the trajectory.

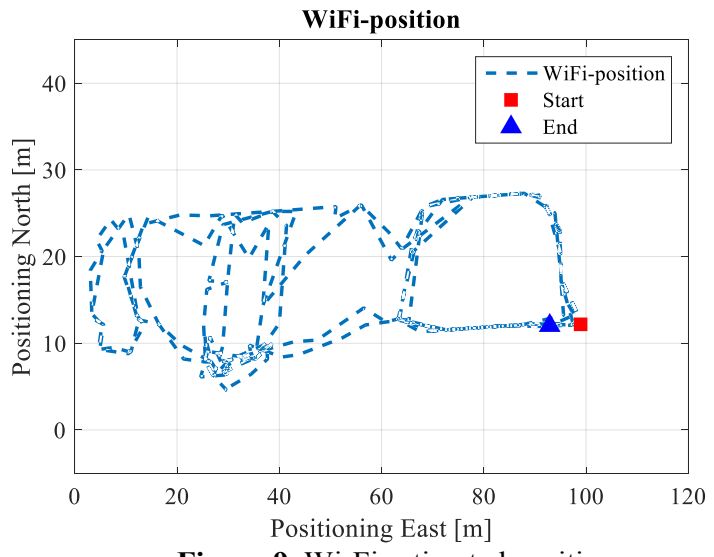

Figure 9. Wi-Fi estimated position

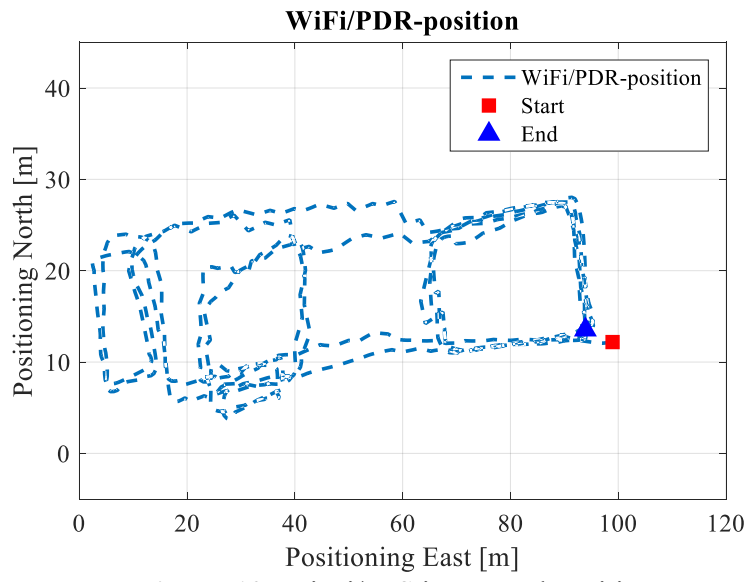

Figure 10. Wi-Fi/INS integrated position

Figure 11 is a Map/Wi-Fi/INS integrated position using the APF-based Map aiding method. By comparing Figure 11 with the reference trajectory in Figure 7, it is obvious that the estimated trajectory almost matches the reference trajectory. 
Additionally, when we combine the Map matching method with the Map aiding method, the user's trajectory will further convergence, the RMS error improve 1 meter when MM method is applied.

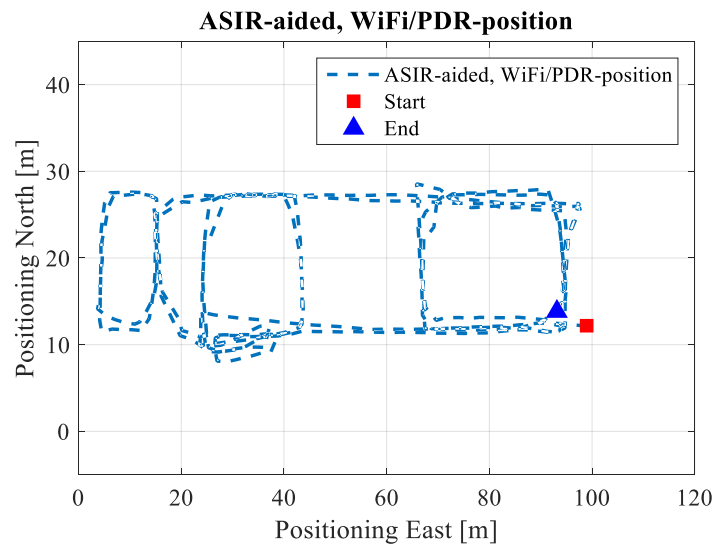

Figure 11. Map/INS/Wi-Fi integrated position using map

\section{CONCLUSION}

An indoor map aided INS/Wi-Fi integrated LBS is proposed on smartphone platform. Three modules are combined through a two-layer Kalman filter/Particle filter structure algorithm. The two-layer structure take advantage of both APF and KF, ensuring the inertial sensor data utilization rate while reducing the update frequency of APF. By performing experiments, we can easily obtain the following conclusions: the INS/Wi-Fi integrated algorithm can improve the accuracy of the lower filter INS solution. In addition, the MA-based APF method can dramatically improve the accuracy of the INS-derived position solution. Moreover, by adding the MM algorithm, the MAbased APF results were further optimized. This research makes valuable use of free ubiquitous Wi-Fi signal and indoor map information, and apply them to correct the INS accumulative errors. This can decrease the cost of LBS and has significant meaning for its popularization.

\section{ACKNOWLEDGEMENTS}

\section{REFERENCES}

Junglas, A., 2008. "Location based services." Communications of the ACM 51.3: 65-69.

Marketsandmarkets, 2016. "Location-Based Services (LBS) and Real Time Location Systems (RTLS) Market by Location (Indoor and Outdoor), Technology (Context Aware, UWB, $B T / B L E$, Beacons, A-GPS), Software, Hardware, Service and Application Area - Global Forecast to 2021". http://www .marketsandmarkets.com/Market-Reports/location-basedservicemarket96994431.html?gclid=CN2qtPLviNICFcePswodd OIHOw

Asghar, Dr, Muhammad Zubair, 2014. "A Review of Location Technologies for Wireless Mobile Location-Based Services." Journal of American Science 10.7: 110-18.
Lan, Haiyu, 2015. "A novel kalman filter with state constraint approach for the integration of multiple pedestrian navigation systems." Micromachines 6.7: 926-952.

Naser, El-Sheimy, 2012. "Inertial techniques and application, ENGO 623-course notes". Department of Geomatics Engineering, University of Calgary.

Cho, S. Y. 2006. "MEMS based pedestrian navigation system". Journal of navigation, 59, 135-153.

Niu, X., 2012. "Observability analysis of non-holonomic constraints for land-vehicle navigation systems." J. Glob. Position. System 11: 80-88.

Yu, Chunyang, et al. "A Map/INS/Wi-Fi Integrated System for Indoor Location-Based Service Applications." Sensors 17.6 (2017): 1272.

Bahl, Paramvir, 2000. "RADAR: An in-building RF-based user location and tracking system." INFOCOM 2000. Nineteenth Annual Joint Conference of the IEEE Computer and Communications Societies. Proceedings. IEEE. Vol. 2. Ieee.

Stella, Maja, 2014. "Fingerprinting based localization in heterogeneous wireless networks." Expert systems with applications 41.15: 6738-6747

Lan, Haiyu, 2015. "An integrated PDR/GNSS pedestrian navigation system." China Satellite Navigation Conference (CSNC) 2015 Proceedings: Volume III. Springer Berlin Heidelberg.

Gustafsson, Fredrik, 2010. "Particle filter theory and practice with positioning applications." IEEE Aerospace and Electronic Systems Magazine 25.7: 53-82.

Yu, Chunyang, et al. "Map-Based Indoor Pedestrian Navigation Using an Auxiliary Particle Filter." Micromachines 8.7 (2017): 225 .

Chunyang Yu,2016. "Particle Filter for Indoor Map-Aided Navigation: Trade Off between Estimation Accuracy and Computational Speed". ION- (PTTI) 2016 conference, Monterey, California.

Grisetti, G.,2007. "Improved techniques for grid mapping with rao-blackwellized particle filters". Robotics, IEEE Transactions, 23, 34-46.

Zanella, Andrea, 2014. "Internet of things for smart cities." IEEE Internet of Things Journal 1.1: 22-32.

Cho, S. Y, 2006. "MEMS based pedestrian navigation system. Journal of navigation", 59, 135-153.

Zhang, X., 2012. "Observability analysis of non-holonomic constraints for land-vehicle navigation systems". Journal of Global Positioning Systems, 11, 80-88. 